\title{
Auditory Cortical Local Subnetworks Are Characterized by Sharply Synchronous Activity
}

\author{
Craig A. Atencio and Christoph E. Schreiner \\ Coleman Memorial Laboratory, Department of Otolaryngology-HNS, The UCSF Center for Integrative Neuroscience, University of California, San Francisco, \\ California 94143-0444
}

In primary auditory cortex (AI), broadly correlated firing has been commonly observed. In contrast, sharply synchronous firing has rarely been seen and has not been well characterized. Therefore, we examined cat AI local subnetworks using cross-correlation and spectrotemporal receptive field (STRF) analysis for neighboring neurons. Sharply synchronous firing responses were observed predominantly for neurons separated by $<150 \mu \mathrm{m}$. This high synchrony was independent of layers and was present between all distinguishable cell types. The sharpest synchrony was seen in supragranular layers and between regular spiking units. Synchronous spikes conveyed more stimulus information than nonsynchronous spikes. Neighboring neurons in all layers had similar best frequencies and similar STRFs, with the highest similarity in supragranular and granular layers. Spectral tuning selectivity and latency were only moderately conserved in these local, high-synchrony AI subnetworks. Overall, sharp synchrony is a specific characteristic of fine-scale networks within the AI and local functional processing is well ordered and similar, but not identical, for neighboring neurons of all cell types.

\section{Introduction}

Across fields and layers, auditory cortical neurons exhibit correlated activity of low magnitude (cross-correlation coefficients $<0.1$ ) and low temporal precision (correlation widths $>15 \mathrm{~ms}$; Brosch and Schreiner, 1999; Eggermont, 2000; Atencio and Schreiner, 2010a). Seldom observed, however, are corticocortical neuron pairs with high synchrony, characterized by tightly coordinated firing (correlation widths $<3$ ms; Eggermont, 2000). This is surprising because high discharge synchrony has been observed in other sensory cortices and primary auditory cortex (AI) shares many structural and functional similarities with these systems (Swadlow et al., 1998; Swadlow, 2003; Douglas and Martin, 2004).

Synchrony is supported by multiple aspects of cortical circuitry. Sensory cortices are composed of a dense matrix of interconnected excitatory and inhibitory neurons (Fino and Yuste, 2011; Packer and Yuste, 2011). Within the matrix, the connectivity between neighboring neurons appears to be indiscriminate (Stepanyants et al., 2008), is greatest at short distances (Holmgren et al., 2003; Boucsein et al., 2011; Packer and Yuste, 2011), and is independent of species (Bannister and Thomson, 2007).

Received May 13, 2013; revised 0ct. 11, 2013; accepted 0ct. 16, 2013.

Author contributions: C.A. and C.E.S. designed research; C.A. and C.E.S. performed research; C.A. analyzed data; C.A. and C.E.S. wrote the paper.

This work was supported by the National Institutes of Health (Grants DC02260 and DC011874), the Coleman Memorial Fund, and Hearing Research Inc. (San Francisco, CA). We thank Andrew Tan, Marc Heiser, Kazuo Imaizumi, and Benedicte Philibert for experimental assistance and Mark Kvale for the use of his SpikeSort 1.3 Bayesian spikesorting software.

The authors declare no competing financial interests.

Correspondence should be addressed to Craig A. Atencio, Coleman Memorial Laboratory, Department of Otolaryngology-HNS, The UCSF Center for Integrative Neuroscience, University of California, 675 Nelson Rising Lane, San Francisco, CA 94143-0444. E-mail: craig@phy.ucsf.edu.

DOI:10.1523/JNEUROSCI.2014-13.2013

Copyright $\odot 2013$ the authors $\quad 0270-6474 / 13 / 3318503-12 \$ 15.00 / 0$
Further, the many classes of connected excitatory and inhibitory cells allows for a large diversity of connections within a layer (Thomson and Lamy, 2007; Otsuka and Kawaguchi, 2009). Auditory cortex also contains these connectivity features and might exhibit high synchrony between local network elements.

Different mechanisms underlying high synchrony have been proffered. Thalamocortical networks may provide fast feedforward excitation to layer 4 (Blomquist et al., 2009) and by thalamic neurons that directly target inhibitory interneurons such as parvalbumin (PV) interneurons (Thomson and Lamy, 2007). In turn, PV cells receive fast input from pyramidal cells within the same layer (Thomson and Lamy, 2007). Through these reciprocal connections, high synchrony could result. In addition, electrical coupling between PV neurons may contribute to synchronous firing (Galarreta and Hestrin, 1999; Gibson et al., 1999). Therefore, an analysis of local synchrony needs to consider layer and cell type identity.

Because synchrony is largely a consequence of both the connectivity between local cortical neurons and the contribution of tightly aligned common input, it is important to characterize the functional processing within these local circuits. Receptive fields in dense local networks may be diverse (Smith and Häusser, 2010). For example, in visual cortex, spatial frequency is not clearly clustered within local pools of neurons (Molotchnikoff et al., 2007) and, at least in upper layers of mouse auditory cortex, tonotopy is present at the macroscale, but seems diminished at microscales (Bandyopadhyay et al., 2010; Rothschild et al., 2010). Therefore, at least in rodents, fine-scale networks may contain a diverse range of receptive field characteristics.

We characterized functional connectivity and receptive fields of local neighboring neurons in cat AI columns. Constituent neurons of local subnetworks were isolated from a single contact in a linear multielectrode array. We related the observed synchrony to 
local receptive field diversity and observed high timing precision that was limited to neurons in close spatial proximity, suggesting that synchrony may be a sign for enhanced local integration, encoding fidelity, and the efficient transfer of auditory information to synaptic targets.

\section{Materials and Methods}

Surgical procedures, stimulation, and recording. All experimental procedures were approved by the University of California-San Francisco Committee for Animal Research under protocol AN086113-01B. The experimental procedures used in this study have been described previously (Atencio and Schreiner, 2010a, 2010b). Briefly, young female ( $n=$ $8)$ and male $(n=2)$ adult cats were given an initial dose of ketamine (22 $\mathrm{mg} / \mathrm{kg})$ and acepromazine $(0.11 \mathrm{mg} / \mathrm{kg})$ and then anesthetized with pentobarbital sodium (Nembutal, $15-30 \mathrm{mg} / \mathrm{kg}$ ) during the surgical procedure. The animal's temperature was maintained with a thermostatic heating pad. Bupivacaine was applied to incisions and pressure points. Surgery consisted of a tracheotomy, reflection of the soft tissues of the scalp, craniotomy over AI, and durotomy. After surgery, to maintain an areflexive state, the animal received a continuous infusion of ketamine/ diazepam (2-5 mg/kg/h ketamine, $0.2-0.5 \mathrm{mg} / \mathrm{kg} / \mathrm{h}$ diazepam in lactated Ringer's solution).

With the animal inside a sound-shielded anechoic chamber (IAC), stimuli were delivered via a closed speaker system to the ear contralateral to the exposed cortex (electrostatic diaphragms; Stax). Extracellular recordings were made using multichannel silicon recording probes provided by the University of Michigan Center for Neural Communication Technology. The probes contained 16 linearly spaced recording channels, with each channel separated by $150 \mu \mathrm{m}$. We only used probes with channel impedances between 2 and $3 \mathrm{M} \Omega$, because these impedances allowed us to resolve single units. Probes were carefully positioned orthogonally to the cortical surface and lowered to depths between 2300 and $2400 \mu \mathrm{m}$ using a microdrive (David Kopf Instruments).

Neural traces were band-pass filtered between 0.6 and $6 \mathrm{kHz}$ and recorded to disk with a Neuralynx Cheetah A/D system at sampling rates between 18 and $27 \mathrm{kHz}$. After each experiment, the traces were sorted offline with a Bayesian spike-sorting algorithm (Lewicki, 1994, 1998). Only those events in the traces that exceeded the DC baseline by five root mean square noise levels were used in the spike-sorting procedure (termed spike events). Most channels of the probe yielded 1-2 well isolated single units. Bayesian spike sorting allows the proper classification of spikes even if they overlap with an action potential of another neuron. When a spike event cannot be assigned to a single unit, an overlap decomposition procedure is performed. To resolve a possible overlap, the algorithm uses the single unit waveforms of the neurons on a channel ( $n=2$ for a pair). The two waveforms are summed for different temporal relationships, resulting in a set of summed waveforms. Each sum in the set corresponds to one temporal relationship. This set is then compared with the actual overlap event. If there is a significant fit between one of the waveform sums and the possible overlap, then the spike times of the two units are recorded. Detailed mathematical explanations of this procedure have been described previously (Lewicki, 1994). All recording locations were in $\mathrm{AI}$, as verified through initial multiunit mapping and determined by the layout of the tonotopic gradient and bandwidth modules on the crest of the ectosylvian gyrus (Imaizumi and Schreiner, 2007). After spike sorting, the action potential waveforms were classified using established methods (Bruno and Simons, 2002; Atencio and Schreiner, 2008). The duration of each spike waveform was calculated and neurons with spike durations $<0.6 \mathrm{~ms}$ were classified as fast-spiking units (FSUs), whereas those with durations $>0.7 \mathrm{~ms}$ were classified as regular spiking units (RSUs). Pairwise analysis was restricted to neurons that were identified from a single electrode contact and did not appear on neighboring contacts (spacing of contacts: $150 \mu \mathrm{m}$ ); therefore, their spatial separation was likely $<150 \mu \mathrm{m}$.

Stimulus. For each recording site, pure tones were presented in a random sequence. The amplitudes and frequencies of the tones spanned $0-70 \mathrm{~dB}(5 \mathrm{~dB}$ steps $)$ and $2.5-40 \mathrm{kHz}$ (0.1 octave [oct] steps), respectively. All neurons were also probed with a broadband $(0.5-40 \mathrm{kHz})$ dynamic moving ripple (DMR) stimulus (Escabi and Schreiner, 2002; Atencio et al., 2008). The maximum spectral modulation frequency of the DMR was 4 cycles/oct, and the maximum temporal modulation frequency was 40 cycles/s (Escabi and Schreiner, 2002). The maximum modulation depth of the spectrotemporal envelope was $40 \mathrm{~dB}$. Mean intensity was set at $30-50 \mathrm{~dB}$ above the average pure tone threshold.

Connectivity. To analyze the functional connectivity between neurons, we followed standard cross-covariance procedures (Rosenberg et al., 1989; Halliday and Rosenberg, 1999). First, spike trains were obtained by binning the spike times with $0.5 \mathrm{~ms}$ resolution. For a single spike train, $A(n)$, $n$ is the bin number and $A(n)$ is either 1 (spike) or 0 (no spike). For two spike trains, $A(n)$ and $B(n)$, the mean intensities, $P_{A}$ and $P_{B}$, for a sample of duration $D$ bins, are estimated as $P_{A}=N_{A} / D$ and $P_{B}=N_{B} / D$, where $N_{A}$ and $N_{B}$ are the total number of spikes in trains $A$ and $B$, respectively. For the spike trains in this study, the stimulus duration was either 15 or 20 min, giving $D=$ $1,800,000$ or $2,400,000$ bins.

The cross-correlation function, or correlogram, for spike trains $A(n)$ and $B(n)$ is then estimated as follows:

$$
C_{A B}(m)=\sum_{n=0}^{D-m} A(n+m) B(n)
$$

From $C_{A B}(m)$, an unbiased estimate of the second order cross-product density, $P_{A B}(m)$, is calculated as follows:

$$
P_{A B}(m)=\frac{C_{A B}(m)}{\Delta \cdot D}
$$

where $\Delta$ is the bin size of the spike train in milliseconds. The crosscovariance function, $Q_{A B}(m)$, is then defined as follows:

$$
Q_{A B}(m)=P_{A B}(m)-P_{A} \cdot P_{B}
$$

Therefore, the cross-covariance function is a scaled version of the crosscorrelation function with the mean background activity removed. This allows excitatory and suppressive interactions to be visualized more easily. Cross-covariance values that are approximately zero represent chance coincidences between the two spike trains. Deflections from zero represent how the activity of one neuron influences the firing of the other neuron. Note that $\lim _{|m| \rightarrow \infty} Q_{A B}(m)=0$. The cross-covariance function $Q_{A B}(m)$ has an asymptotic distribution from which its variance can be estimated (Halliday and Rosenberg, 1999). Under the assumption of independent Poisson spike trains, the variance of $Q_{A B}(m)$ may be approximated as follows:

$$
\operatorname{Var}\left(Q_{A B}(m)\right) \cong \frac{P_{A} \cdot P_{B}}{\Delta \cdot D}
$$

Therefore, for two spike trains, upper and lower $99 \%$ confidence limits $(\mathrm{CL})$ for $Q_{A B}(m)$ can be set as follows:

$$
C L= \pm 3\left(\frac{P_{A} \cdot P_{B}}{\Delta \cdot D}\right)^{1 / 2}
$$

Only cross-covariance functions with two consecutive bins satisfying the 99\% CLs were analyzed in this study. For some neuron pairs, shift predictors were also estimated (Fig. $1 D, F$, gray curves). As noted previously, shift predictors did not approach the magnitude of cross-covariance functions, indicating that the observed functional correlation is not merely due to stimulus synchronization (Atencio and Schreiner, 2010a).

Two quantities were used to estimate the strength and degree of synchrony. To estimate the strength of functional connections, we calculated a normalized cross-correlation coefficient (CCC; Agmon, 2012). For each correlogram, $C_{A B}$, the CCC was defined as follows:

$$
C C C=\left[C_{A B}(\mathrm{PD})-\frac{N_{A} N_{B}}{D}\right]\left(\min \left(N_{A}, N_{B}\right)-\frac{N_{A} N_{B}}{D}\right)
$$

where the peak delay $(\mathrm{PD})$ is the time at which the peak in the correlogram occurs, $C_{A B}(\mathrm{PD})$, is the number of spikes in the correlogram at the 
A

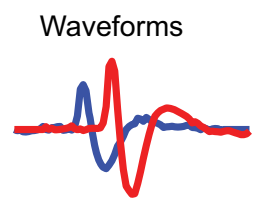

B
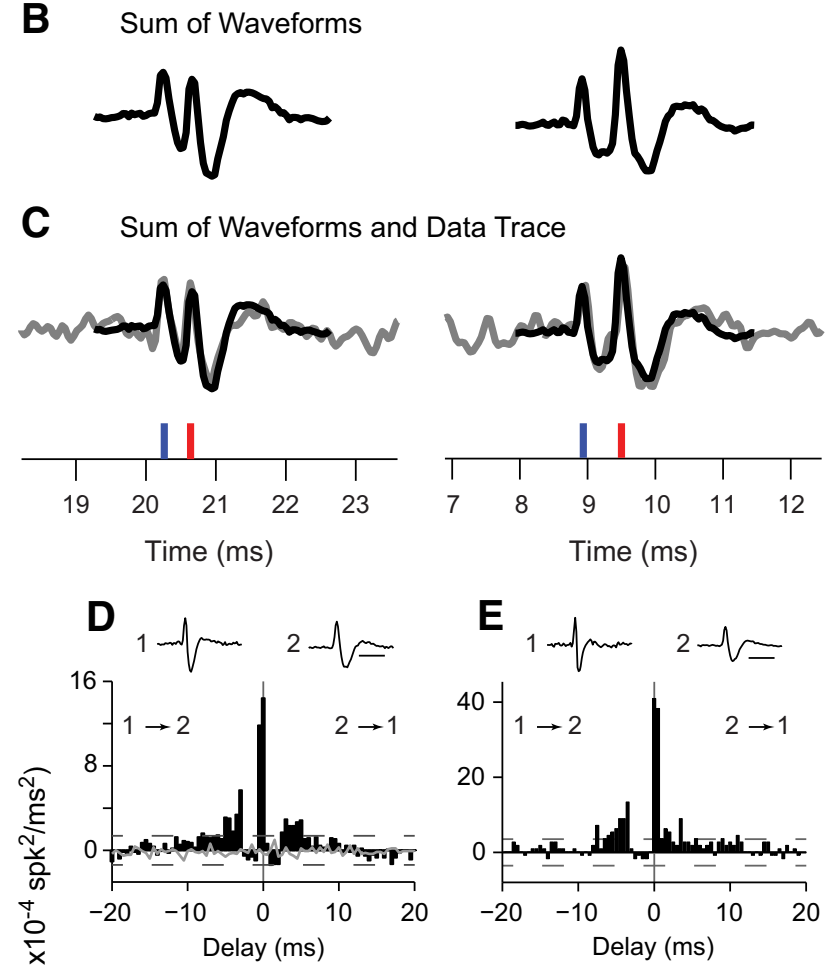

\section{$F$}
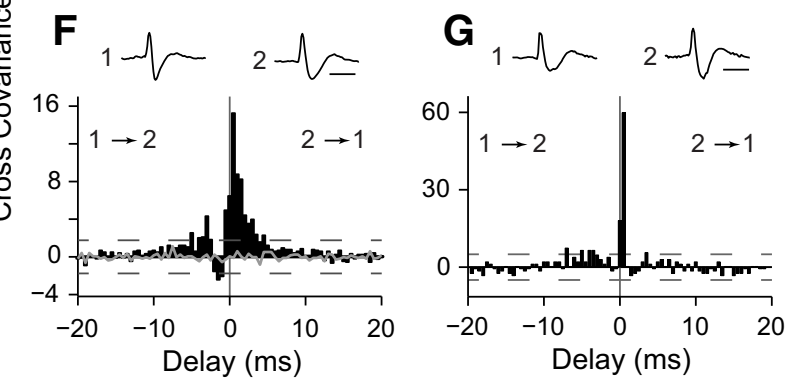

Figure 1. Waveform and connectivity analysis of pairs of neurons. $A-C$, Each column shows spike waveforms for a pair of recorded neurons. $\boldsymbol{A}$, Waveforms for the two pairs. $\boldsymbol{B}$, The sum of the two waveforms in $\boldsymbol{A}$. $C$, The sum of the waveforms in $\boldsymbol{B}$ superimposed on the recorded neural trace. The estimated spike times for the pairs are shown below by the blue and red bars. $\mathbf{D}-\mathbf{G}$, Example cross-covariance functions. Waveforms for the two constituent neurons are shown in the plot. Scale bar, $1 \mathrm{~ms}$. The direction of information flow in the cross-covariance function is shown by the arrow from one waveform to the other. Dashed lines in $\boldsymbol{D}-\mathbf{G}, 99 \%$ confidence intervals. Solid lines in $\boldsymbol{D}, \boldsymbol{F}$, shift predictors.

peak delay, and $\min \left(N_{A}, N_{B}\right)$ is the number of spikes in the shorter spike train. This form of the CCC is impervious to firing rate differences between the spike trains of the two neurons.

Spectrotemporal receptive fields. Data analysis was performed in MATLAB (MathWorks). For each neuron, the spike-triggered average was used to estimate the spectrotemporal receptive field (STRF; Escabi and Schreiner, 2002). STRFs were thresholded so that only significant features $(p<0.01)$ were included in the analysis.

Basic parameters: best frequency, spectral tuning, and latency. The best frequency (BF) was extracted by summing the STRF across time and then identifying the frequency corresponding to the maximum value. Latency was obtained in a similar fashion: the STRF was summed across fre- quency and then the time at which the maximum occurred was calculated. The bandwidth (BW) of each STRF was obtained by first setting all negative values in the STRF to zero. Then, the main excitatory subfield frequency boundaries were estimated as the frequencies at which the STRF magnitude decreased to $10 \%$ of the peak STRF value. Spectral tuning was then defined as the quality factor $(Q)$, and calculated as follows: $Q=\mathrm{BF} / \mathrm{BW}$.

Bicellular information analysis. The information transmitted by synchronous, or bicellular, spikes was estimated using receptive field analysis. Bicellular spikes were obtained from the peak in the cross-covariance function. To identify the spikes in the peak, the half-width (HW) of the cross-covariance function was estimated, where HW was the width of the function at half of the peak amplitude. The window that was used to obtain bicellular spikes extended from $1 \mathrm{HW}$ below the peak delay to 1 $\mathrm{HW}$ above the peak delay value (window $=[\mathrm{PD}-\mathrm{HW}, \mathrm{PD}+\mathrm{HW}])$. We then estimated the STRF for the bicellular spike train, resulting in three STRFs, one for each neuron in a pair and one for the bicellular spikes.

We then estimated the information conveyed by each STRF using previous methodologies (Atencio et al., 2008). First, each stimulus segment, $s$, that preceded a spike was correlated with the STRF by projecting it onto the STRF via the inner product $z=s \cdot S T R F$. These projections form the probability distribution $P(z \mid s p i k e)$. We then formed the prior probability distribution, $P(z)$, by projecting a large number of randomly selected stimulus segments onto the STRF. We next calculated the mean and SD of $P(z), \mu$, and $\sigma . P(z \mid s p i k e)$ and $P(z)$ were transformed to units of SD via $x=(z-\mu) / \sigma$ to obtain the distributions $P(z \mid s p i k e)$ and $P(x)$.

The mutual information between projections onto an STRF, $v$, and single spikes was computed according to

$$
I(v)=\int d x P_{v}(x \mid \text { spike }) \log _{2}\left[\frac{P_{v}(x \mid \text { spike })}{P_{v}(x)}\right]
$$

Information was only estimated if there were at least 200 spikes in the bicellular spike train. For the bicellular spikes, all spikes were used. We corrected for the number of spikes because there were fewer bicellular spikes than spikes in either of the original spike trains. For each original spike train, a subset of spikes equal to the number of spikes in the bicellular spike train was randomly sampled without replacement. Information was estimated for this subset of spikes. This resampling process was repeated 50 times to obtain a mean information value for each original STRF.

Each information value was calculated using different fractions of the dataset for each spike train. To accomplish this, the information values were calculated over the first $90,92.5,95,97.5$, and $100 \%$ of the test dataset. The information calculated from these data fractions was plotted against the inverse of the data fraction percentage (1/90, 1/92.5, etc.). We extrapolated the information values to infinite dataset size by fitting a line to the data and taking the ordinate intersect as the information value for unlimited data size. Therefore, this procedure resulted in three information values: one for the bicellular spikes, and one each for the original spike trains.

Comparisons between neurons. Throughout this study, we compared functional processing for each member in a pair of neurons. To provide a principled way in which to plot the values for each pair, the neuron with the shortest spike waveform had its value plotted on the abscissa, whereas the neuron with the longer waveform had its value plotted on the ordinate. For parameter differences, the absolute value of either the difference or the oct difference was used. The similarity between STRFs was measured using the Pearson correlation coefficient. For laminar analyses, layers were defined as described previously: Supragranular (Supra: 0-600 $\mu \mathrm{m}$ ), Granular (Gran: 700-1100 $\mu \mathrm{m}$ ), and Infragranular (Infra: 1200-2000 $\mu \mathrm{m}$; Atencio and Schreiner, 2010b). Population statistics are presented as mean \pm SD unless otherwise noted.

\section{Results}

Synchronous activity is characterized by time-locked spiking between neurons. Tightly locked spiking is present when spikes from one neuron occur in close temporal relationship with the 
spikes from another neuron. Outside of this time window, spikes from the neurons remain essentially uncoordinated. How can we detect this synchrony in cortex? We examined functional processing within fine-scale networks by recording from pairs of neurons at the same electrode contact location $(N=670)$. We used 16-channel recording probes that spanned all layers of AI. Neuron pairs provide a window into the function of local subnetworks within AI due to their close geometric proximity. Neurons in each pair were differentiated using unique features of their spike waveforms (Fig. 1A-C).

For each pair of neurons, we estimated the functional connectivity through cross-covariance analysis. Previous recordings showed that the same neuron was not recorded from adjacent probe recording channels; therefore, the neurons in a pair were likely within $150 \mu \mathrm{m}$ of each other (Atencio and Schreiner, 2010a). Analyses of the spike waveform shape allowed us to identify functional connectivity between different and identical cell types, such as neurons with a fast, or thin, spike waveform (FSUs) or neurons with a longer, or regular, spike waveform (RSUs; Fig. $1 D, E$; spike duration noted below spike waveform). The receptive field properties of FSUs and RSUs were consistent with those from earlier studies (Atencio and Schreiner, 2008; Wu et al., 2008). We also found functional connectivity between pairs of neurons with the same type of waveform (see Fig. $1 F, G$ for RSUs). In cross-covariance functions, the delay at which the peak occurs, the PD, indicates the relative timing difference between the spiking of neurons. It reflects either relative common-input timing or the flow of information from one neuron to the other. PDs centered at $0 \mathrm{~ms}$ delay indicate that the two neurons fire simultaneously. For many pairs, the covariance function had PDs $<4 \mathrm{~ms}$, indicating highly synchronous firing. The HW of the covariance function (width of the function at half the peak amplitude) indicates the precision of the synchrony. Broad covariance functions (HWs $>15 \mathrm{~ms}$ ), such as those that we previously reported between neurons across different layers (Atencio and Schreiner, 2010a) or those between different cortical fields (Eggermont, 2000), indicate general synchronization, but not very precisely coordinated firing. For neuron pairs in close spatial proximity, we found that the majority had half-widths that were $<3 \mathrm{~ms}$, indicating that the synchrony among colocalized neurons was consistently sharp (Fig. 2D).

On average, the peak in the cross-covariance function occurred within only a few milliseconds ( $1.84 \pm 2.63 \mathrm{~ms}$; Fig. $2 A)$, indicating the close temporal proximity of spiking for the average event. Short PDs can also be found in broad functional connectivity, so synchrony must be further evaluated with respect to the width of the cross-covariance function. The HWs of the cross-covariance functions were extremely narrow, usually $<2 \mathrm{~ms}(1.64 \pm 1.16$; Fig. $2 D)$, indicating that the occurrence of spikes in the two neurons is not only at close temporal proximity but also has low variance. This relative coordinated spiking precision is approximately one order of magnitude better than the HWs for interlaminar correlations (Atencio and Schreiner, 2010a) and for correlations along horizontal isofrequency contours (Brosch and Schreiner, 1999). The strength of the connection, as estimated from the CCC was on average $0.084 \pm$ 0.126 (Fig. $2 G$ ), indicating that $\sim 8 \%$ of the spikes occur at the timing given by the peak delay. These CCCs are higher than mean values previously found for intracortical or interlaminar correlations (Atencio and Schreiner, 2010a). Therefore, over the population of neuron pairs, sharp synchrony is present between nearby auditory cortical neurons.

Is sharp synchrony dependent on cortical layer? To assess this, we grouped our data into three layers: Supra $(n=50), \operatorname{Gran}(n=$
202), and Infra $(n=294)$ as per Atencio and Schreiner (2010b). We found sharp synchrony in all layers of auditory cortex. Peak delays were $<2 \mathrm{~ms}$ in all layers (Fig. $2 B$ ), with no statistical difference between the layers (Supra PD: $2.29 \pm 3.87 \mathrm{~ms}$; Gran: $1.76 \pm 2.32 \mathrm{~ms}$; Infra: $1.66 \pm 2.28 \mathrm{~ms}$; Supra vs Gran: $p=0.46$; Supra vs Infra: $p=0.34$; Gran vs Infra: $p=0.92$, rank-sum tests; Fig. 2C). Half-widths were also narrow in each layer (Fig. 2E), again, with no significant differences between the layers (Supra HW: $1.46 \pm 0.85 \mathrm{~ms}$; Gran: $1.55 \pm 0.92 \mathrm{~ms}$; Infra: $1.74 \pm 1.31 \mathrm{~ms}$; Supra vs Gran: $p=0.51$; Supra vs Infra: $p=0.43$; Gran vs Infra: $p=0.79$, rank-sum tests; Fig. $2 F$ ). In each layer, the average HW was $<2 \mathrm{~ms}$. Because the bin widths in the covariance functions were $0.5 \mathrm{~ms}$, this indicates that submillisecond firing precision is present across the cortical thickness. Last, the correlation strength was relatively conserved across layers, with the highest strengths in supragranular layers (Supra CCC: $0.097 \pm 0.110$; Gran: $0.076 \pm$ 0.122; Infra: $0.088 \pm 0.133$; Supra vs Gran: $p=$ 0.0098; Supra vs Infra: $p=0.0183$; Gran vs Infra: $p=0.69$, rank-sum tests; Fig. $2 \mathrm{H}, I)$.

\section{Synchrony between cell types}

Cortex is composed of various excitatory and inhibitory cell types (Markram et al., 2004). Earlier reports found that chemical synapses between fast-spiking inhibitory neurons account for their high and sharp synchrony, as opposed to common input or gap junctions (Galarreta and Hestrin, 2002; Hu et al., 2011). Is synchrony in AI networks dependent on the cell types that compose the local pairs of neurons? To answer this question, we determined whether the spike waveform of a neuron was either from an RSU or an FSU (Bruno and Simons, 2002). RSUs have longer waveforms and are putative excitatory pyramidal neurons. FSUs have shorter spike waveforms and are putative inhibitory basket or chandelier cells (Kawaguchi and Kubota, 1993, 1997). We analyzed the spike waveforms of each neuron and classified them as either RSUs or FSUs. Neurons that did not satisfy the requirements of either group were not included in cell-type analyses.

We identified three types of pairs: FSU-RSU $(n=116)$, RSURSU $(n=376)$, and FSU-FSU $(n=8)$ and estimated the functional connectivity between pairs that comprised these cell classes. All three types of neuron pairs showed similarly narrow cross-covariance functions, with short peak delays between 1 and $3 \mathrm{~ms}$. Specifically, peak delays for FSU-RSU and RSU-RSU celltype pairs appeared slightly shorter, although not significantly, than those for FSU-FSU pairs (FSU-RSU PD: $1.45 \pm 2.30 \mathrm{~ms}$; RSU-RSU: $1.29 \pm 1.34 \mathrm{~ms}$; FSU-FSU: $2.88 \pm 3.34 \mathrm{~ms}$; FSU-FSU vs FSU-RSU: $p=0.24$; FSU-FSU vs RSU-RSU: $p=0.29$; FSURSU vs RSU-RSU: $p=0.29$, rank-sum tests; Fig. $2 J, K$ ).

The half-widths of covariance functions were narrow for all pair types, with most HWs $<2 \mathrm{~ms}$ (Fig. $2 L, M$ ). Of the three cell-type pairs, RSU-RSU pairs had the narrowest HWs, which were slightly but significantly narrower than the HWs for FSURSU pairs (FSU-RSU HW: $1.84 \pm 1.27$ ms; RSU-RSU: $1.60 \pm$ $1.23 \mathrm{~ms}$; FSU-FSU: $2.30 \pm 1.44 \mathrm{~ms}$; FSU-RSU vs RSU-RSU: $p=$ 0.0003; FSU-RSU vs FSU-FSU: $p=0.40$; RSU-RSU vs FSU-FSU: $p=0.065$, rank-sum tests; Fig. $2 M$, inset).

Last, compared with interlaminar and corticocortical correlation strength, CCCs were relatively high for all three cell-type pairs (FSU-RSU CCC: $0.082 \pm 0.095$; RSU-RSU: $0.096 \pm 0.146$; FSU-FSU: $0.061 \pm 0.053$; FSU-RSU vs RSU-RSU: $p=0.96$; FSURSU vs FSU-FSU: $p=0.59$; RSU-RSU vs FSU-FSU: 0.62 , ranksum tests; Fig. 2 N,O; Eggermont, 1992; Atencio and Schreiner, 2010a). Therefore, relatively high and sharp synchrony is present between all different cell types in auditory cortex. Further, al- 

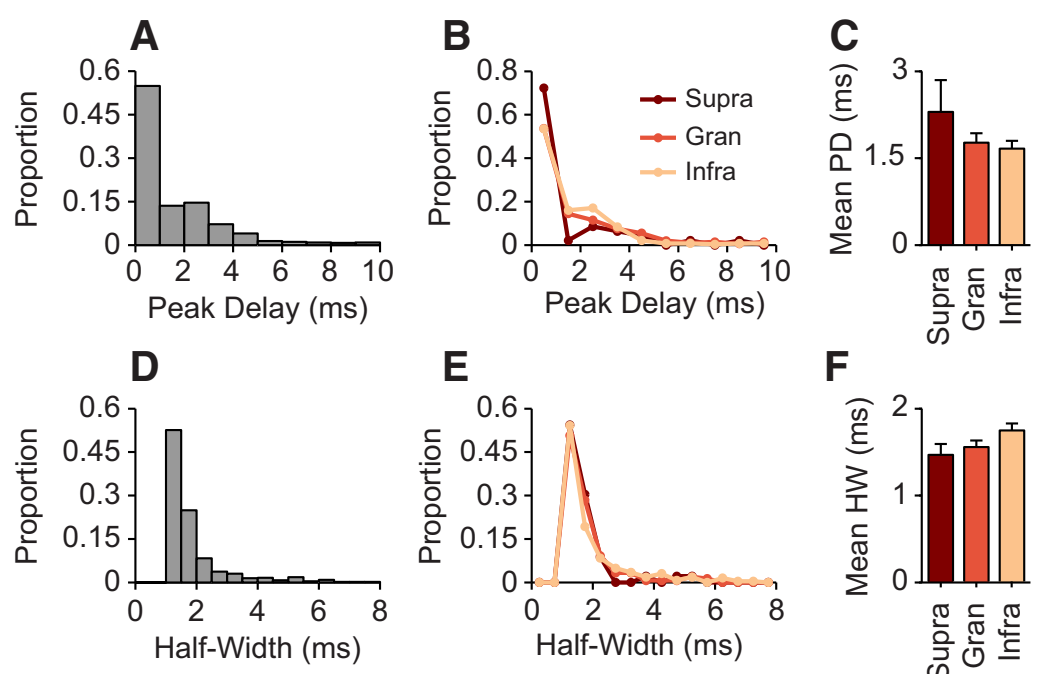

E
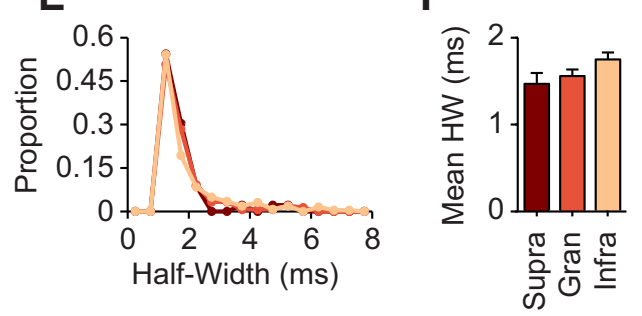

G

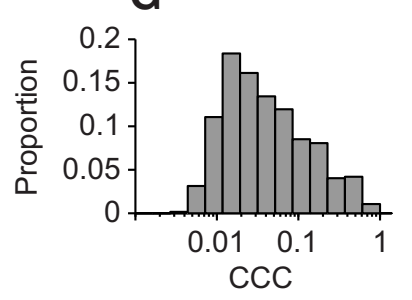

H
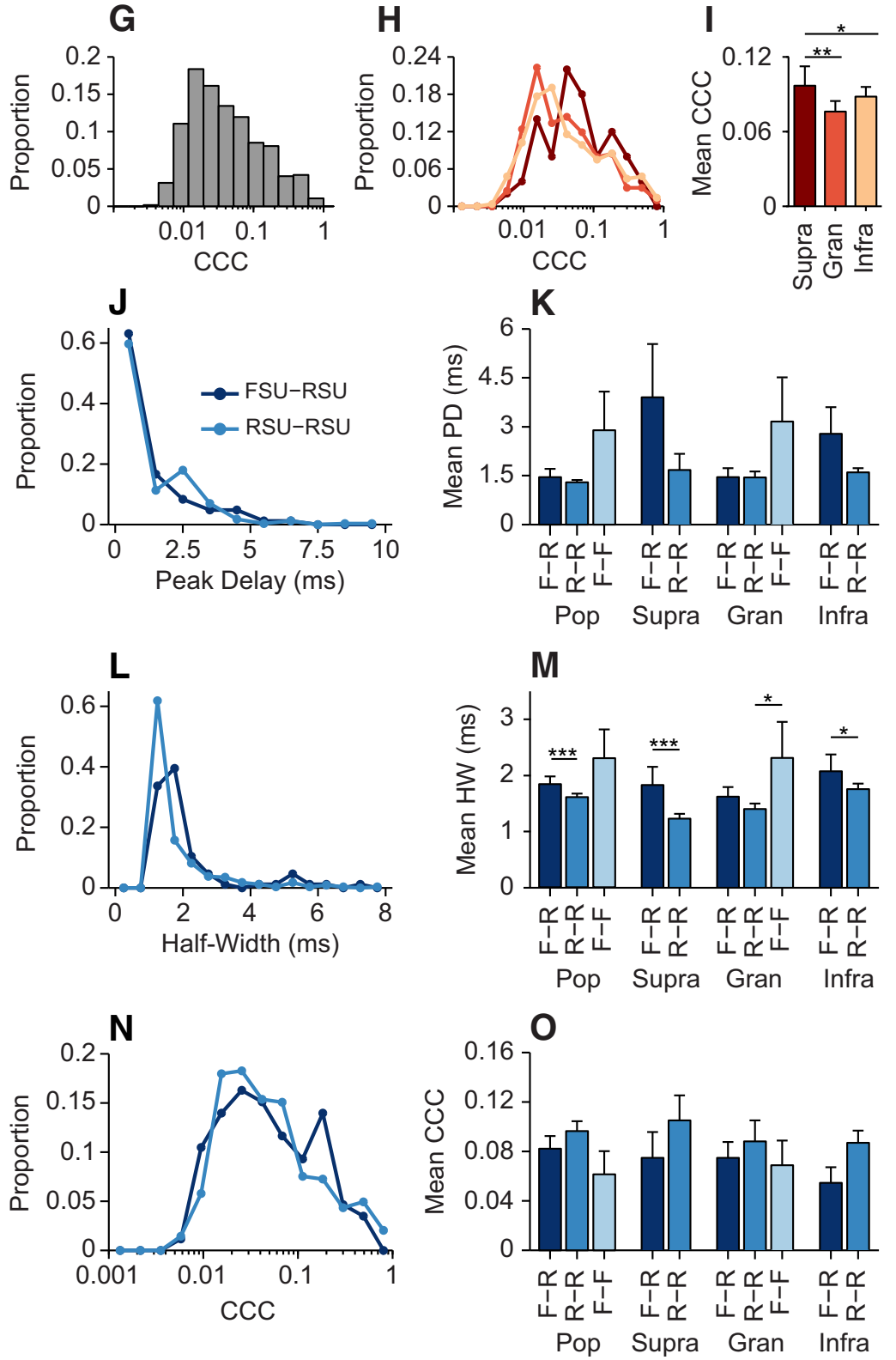

Figure 2. Population, laminar, and cell-type synchrony analysis. $\boldsymbol{A}$, The time at which the peak occurred in cross-covariance functions (PD) was usually $<4 \mathrm{~ms}$, with the majority of peak delays $<2 \mathrm{~ms}$. $\boldsymbol{B}$, PDs were similar in Supra, Gran, and Infra layers. $\boldsymbol{C}$, The mean PDs across layers were $<2 \mathrm{~ms}$, with the shortest PDs in the Gran and Infra layers. $\boldsymbol{D}$, HWs of cross-covariance functions though FSU-FSU submillisecond synchrony has been reported previously ( $\mathrm{Hu}$ et al., 2011), our results show that putative excitatory cell pairs also exhibit this precision.

Cell-type specific synchrony did not vary strongly between cortical layers. Across layers, the peak delays for RSU-RSU pairs were highly conserved ( $p>0.1$, rank-sum tests; Fig. $2 \mathrm{~K}$ ). The PDs for FSU-RSU pairs showed more variability, but even in these cases, the delays were $<4 \mathrm{~ms}$ (Fig. $2 K$ ). The HWs of the pairs were also conserved across layers, with HWs $<2.5 \mathrm{~ms}$ for each type of pair in each layer (Fig. 2M). HWs were narrower for RSU-RSU pairs than for FSURSU pairs in the Supra and Infra layers and narrower than FSU-FSU pairs in Gran layers (Supra FSU-RSU vs RSU-RSU: $p=$ 0.0005; Infra FSU-RSU vs RSU-RSU: $p$ 0.035; Gran FSU-FSU vs RSU-RSU: $p=$ 0.034; all other comparisons: $p>0.05$; rank-sum tests). Finally, the correlation strength was strongest for RSU-RSU pairs in supragranular layers, but the differences were not significant across layers $(p>0.2$, rank-sum tests; Fig. 2O). Likewise, the CCC for FSU-RSU was similar across layers. Because the peak delay, half-width, and CCC of neighboring neurons were similar across layer, the different cell-type pairs share similar synchrony patterns in different parts of the cortical microcircuit.

\section{STRF similarity for pairs}

Does the high synchrony of neighboring neurons imply that their receptive fields are similar? We used spike-triggered STRFs to assess the functional processing properties of each neuron. The STRF jointly characterizes the time and frequency stimulus preferences of a neuron and therefore describes processing dynamics that may be missed with purely spectral or temporal measures. Because the STRF includes joint processing aspects, it compactly and systematically describes stimulus selectivity (Atencio and Schreiner, 2008; Atencio et al., 2008, 2009).

\section{$\leftarrow$}

were usually $<2 \mathrm{~ms}$. $\boldsymbol{E}$, HW distributions across layers were similar. $\boldsymbol{F}$, Mean HWs were consistent across layers, with the smallest HWs in Supra layers. G, CCC was broadly distributed. $\boldsymbol{H}$, Supra pairs of neurons had the highest $C C C$. I I, Mean CCCS were highest in Supra and lowest in granular layers. J, Peak delays for FSU-RSU and RSU-RSU pairs were similar. $\boldsymbol{K}$, Mean peak delays across the population and layer for different pairs of cell types (F-F: FSU-FSU; F-R: FSU-RSU; R-R: RSU-RSU). L, HWs of cross-covariance functions were small for FSU-RSU and RSU-RSU pairs. M, Mean HWs. N, CCCs for FSU-RSU and RSURSU pairs were similar. $\mathbf{0}$, Mean correlation strength for the different pair types. ${ }^{*} p<0.05 ;{ }^{* *} p<0.01 ;{ }^{* * *} p<0.001$. 

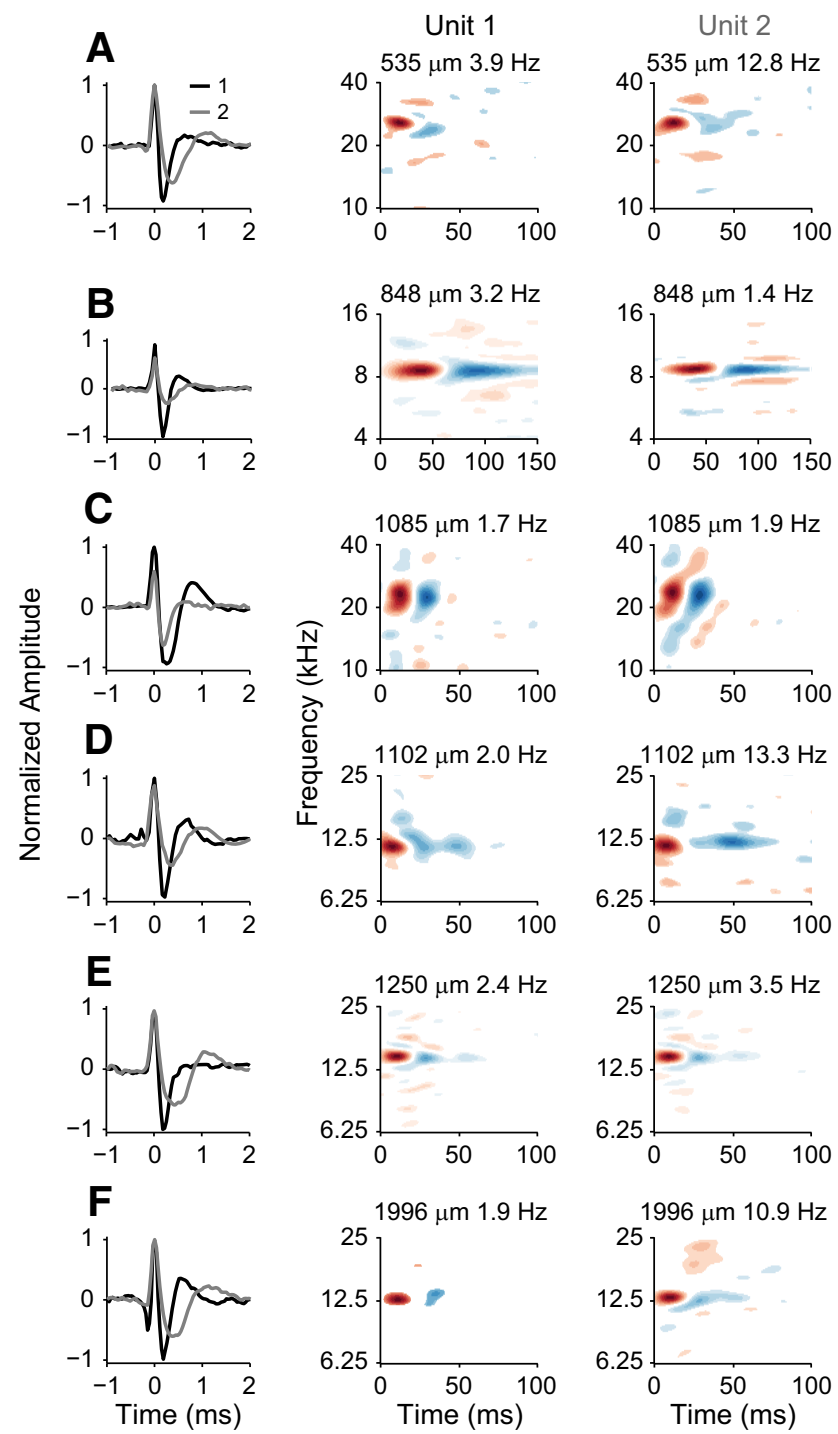

Figure 3. Example waveforms and STRFs. $\boldsymbol{A}-\boldsymbol{F}$, Each row represents two neurons recorded from the same electrode channel. Left: Example neuron spike waveforms. Middle and Right: STRFs corresponding to the spike waveforms. Depth of recording site and the average firing rate of each neuron are indicated above the STRF.

Local pairs of neurons usually had STRFs with moderate to high similarity (Fig. 3). The main aspects of excitatory (Fig. 3, red) and inhibitory (blue) portions of the STRFs were usually congruent, but not necessarily identical. We observed this trend regardless of spike shape.

We compared the global stimulus preferences of each pair of neurons by correlating the STRFs (Fig. $4 A, B$ shows two example comparisons). Across the population, STRF similarity was moderately high (Fig. $4 C$, black curve; $0.50 \pm 0.17$ ). STRF similarity was high in Supra layers and highest in Gran layers (Supra: $0.53 \pm$ 0.16; Gran: $0.56 \pm 0.17$; Infra: $0.43 \pm 0.16$; Fig. $4 C, D$ ). Infra layers had STRFs with the most divergent STRFs (Supra vs Gran: $p=$ 0.19; Supra vs Infra: $p=0.0002$; Gran vs Infra: $p<0.001$, ranksum tests). The magnitude of the values, however, indicated that the excitatory and inhibitory subfield structures were correlated (cf. Fig. 4C,D and $A$ for a similar correlation value). Therefore, at the smallest spatial scales, nearby neurons have globally similar STRFs in all layers, with the most similar STRFs in the Gran and Supra layers.
We also investigated whether high STRF similarities were seen for cell-type pairs. We found that FSU-FSU pairs had the most similar STRFs and that FSU-RSU and RSU-RSU pairs had nearly equivalent distributions (FSU-RSU: $0.51 \pm 0.15$; RSU-RSU: $0.49 \pm 0.18$; FSU-FSU: $0.68 \pm 0.11$; FSU-RSU vs RSU-RSU: $p=$ 0.20; FSU-RSU vs FSU-FSU: $p=0.018$; RSU-RSU vs FSU-FSU: $p=0.01$, rank-sum tests; Fig. $4 E, F)$. When we compared STRF similarity with correlation strength, we found weak trends. STRF similarity for FSU-RSU and FSU-FSU pairs was not significantly correlated with correlation strength (FSU-RSU: $r=0.106, p=$ 0.33; FSU-FSU: $r=-0.064, p=0.8$ ), but RSU-RSU pairs had STRFs that were slightly more similar as the correlation strength increased (RSU-RSU: $r=0.196, p<0.001$ ). We also estimated STRF similarity by shifting the STRFs so that the peak latencies were aligned (Chen et al., 2012). STRF similarities with and without this shift were not statistically different $(p>0.25$, rank-sum test), most likely because pairs of neighboring neurons have latencies that differ by only 1-3 ms. Different cell types throughout local regions of cortex have similar but not identical STRFs.

\section{BF, spectral tuning, and latency across layers}

For nearby neurons, how similar are basic receptive field parameters? The microarchitecture of AI provides a substrate that is used to organize basic receptive field parameters. If the most basic parameters are disorganized, then, despite synchronous activity, subnetworks are likely to represent these parameters in a disorganized manner. Three basic processing parameters were obtained from the STRF: BF, spectral tuning $(Q)$, and latency.

The parameters were similar for the neurons in a pair. Across the population of pairs, BF was highly similar (Fig. 5A) across all frequency ranges, indicating low $\mathrm{BF}$ variance at fine scales throughout AI. BF differences between neurons were minor and were almost entirely $<0.1$ oct $(0.044 \pm 0.097$ oct; median \pm median absolute deviation [MAD]: $0.020 \pm 0.020$ oct; Fig. $5 D$ ). These minor differences were recapitulated across the cortical layers, with pairs in all layers having similar BFs. We observed the smallest differences in supragranular layers and slightly larger differences in infragranular layers, but the laminar distributions were not significantly different (Fig. $5 G$ ).

We then examined the frequency selectivity of each neuron, which is described by spectral tuning (defined as Q; Schreiner, 1991). Spectral tuning displayed modest agreement, indicating either nonidentical spectral integration or the effective convergence of neurons with similar frequency selectivity within local subnetworks (Fig. 5B). The tuning differences were usually within a factor of $1 / 3(0.41 \pm 0.39$ oct; median \pm MAD: $0.31 \pm$ 0.21 oct; Fig. $5 E$ ). We observed the largest differences for pairs in infragranular layers, and smaller differences in granular and supragranular layers (Fig. $5 \mathrm{H}$ ).

Last, the latency of the response, as determined from the peak in the STRF, was highly similar for pairs (Fig. 5C). Latency differences were usually within a few milliseconds $(2.25 \pm 2.37 \mathrm{~ms}$; median \pm MAD: $1.50 \pm 1.00 \mathrm{~ms}$; Fig. $5 F$ ), with the smallest differences in granular layers and the largest in infragranular layers (Fig. 5I). Therefore, within fine-scale AI networks, in all layers, $\mathrm{BF}$ is highly conserved and spectral tuning and latency are moderately conserved.

\section{$\mathrm{BF}$, spectral tuning, and latency for cell type pairs}

Are basic receptive field parameters similar for cell-type pairs? Having identified FSUs and RSUs, we then compared receptive field parameters for the different cell type pairs. BFs were highly correlated for FSU-RSU pairs, as were the BFs for RSU-RSU and 
A
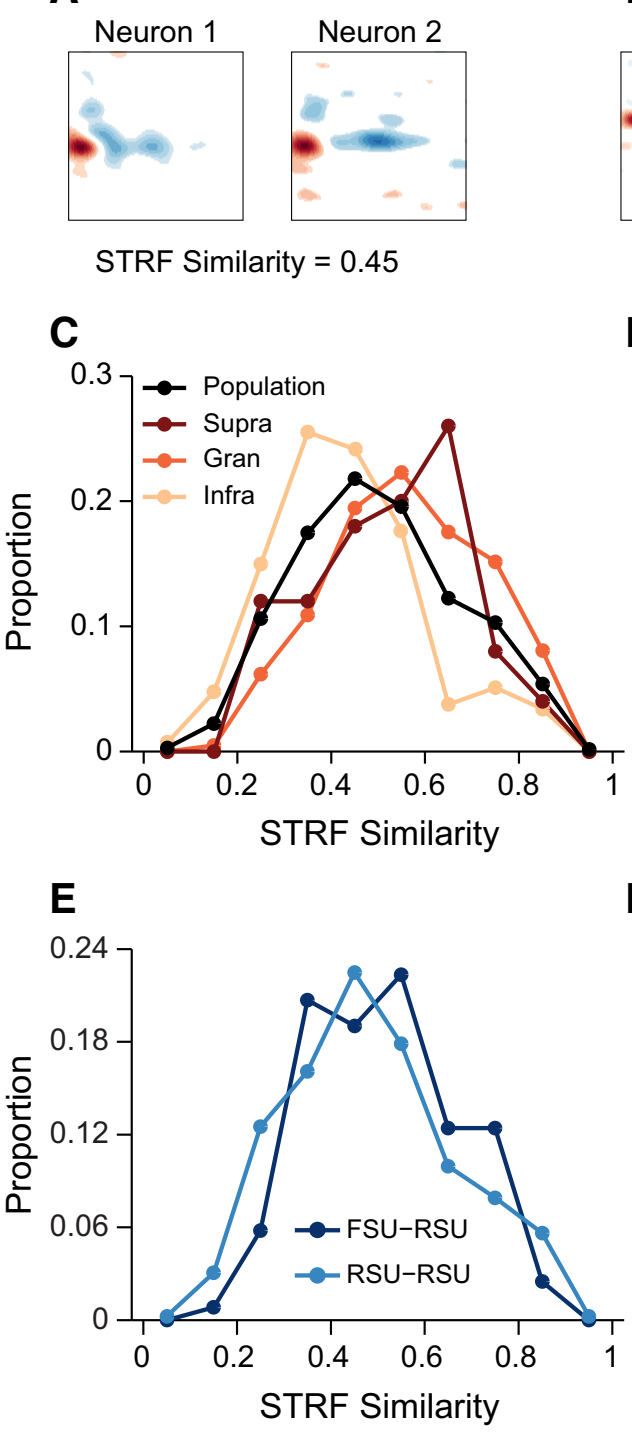

B

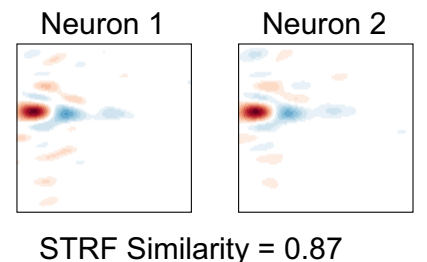

D

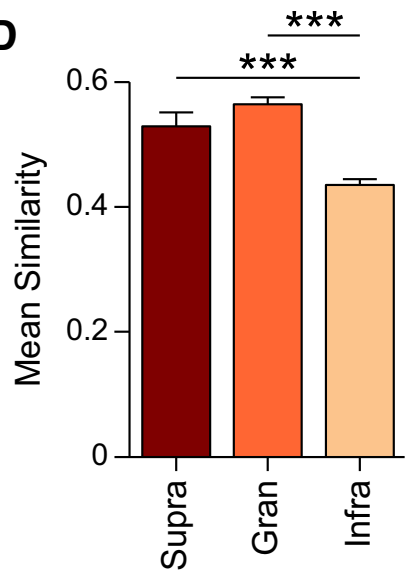

$\mathbf{F}$

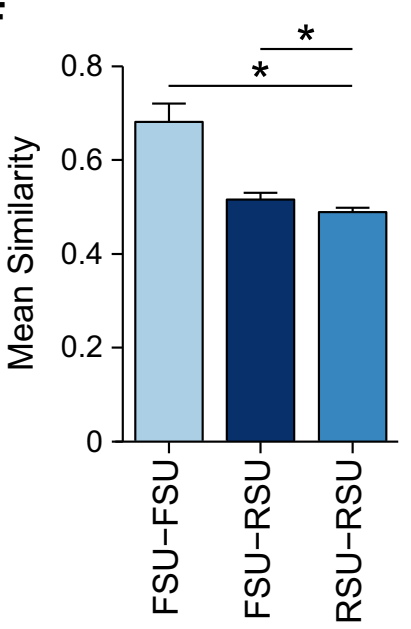

Figure 4. STRF similarity for pairs of neurons. $A, B$, STRFs and STRF similarity for two example pairs of neurons. In $A$, the STRFs are moderately correlated, whereas in $\boldsymbol{B}$, the STRFs are highly correlated. $\boldsymbol{C}$, Population and laminar distributions of STRF similarities. $\boldsymbol{D}$, Mean STRF similarities across layers. $\boldsymbol{E}$, STRF similarity distributions for cell type pairs. $\boldsymbol{F}$, Mean STRF similarity for cell type pairs. ${ }^{*} p<0.05 ;{ }^{* *} p<0.01 ;{ }^{* * *} p<0.001$.

FSU-FSU pairs (FSU-RSU $r=0.992 ; p<0.001$; RSU-RSU $r=$ 0.960, $p<0.001$; FSU-FSU $r=0.690, p=0.058, t$ tests; Fig. $6 A, D, G)$. Spectral tuning was slightly broader for FSUs compared with their RSU partners, whereas it was equally matched for RSU-RSU and FSU-FSU pairs (FSU-RSU pairs: FSU Q: $2.81 \pm 1.31$, RSU Q: $3.21 \pm 1.45 ; p=0.044, t$ test; Fig. $6 B, E, H)$. Last, for FSU-RSU pairs, latency was slightly shorter for FSUs. For the other pair types, latency was similar (FSU-RSU pairs: FSU latency: $12.83 \pm 2.95 \mathrm{~ms}$, RSU latency: $13.71 \pm 3.34 \mathrm{~ms} ; p=$ $0.043, t$ test; Fig. $6 C, F, I)$. These results provide an internal control for our data, because earlier reports also noted that FSUs have broader receptive fields and shorter latencies (Atencio and Schreiner, 2008; Wu et al., 2008).

Receptive field parameter differences between neurons within each cell type pair were generally minor. BF difference was smallest for FSU-RSU pairs $(0.04 \pm 0.05 \mathrm{oct})$, and only FSU-FSU pairs
$(0.10 \pm 0.27 \mathrm{oct})$ were significantly different from FSU-RSU and RSU-RSU pairs $(0.05 \pm 0.12$ oct $)$. Spectral tuning differences were largest for FSU-RSU pairs $(0.46 \pm 0.45 \mathrm{oct})$, which is indicative of the broader tuning that has been observed for FSUs. Last, STRF peak latencies were highly similar between the cell-type pairs, with the smallest differences between neighboring FSUs $(1.38 \pm 0.79 \mathrm{~ms})$. Together, these results show that, within local subnetworks, BF is highly conserved regardless of neuron class and, for FSURSU pairs, FSUs have broader spectral tuning and shorter latencies.

Information gain in local subnetworks Neighboring neurons exhibit sharp synchrony in auditory cortex. Do synchronous spikes signal more information about the stimulus than nonsynchronous spikes? We addressed this by estimating the information that is conveyed by synchronous spikes. First, we estimated the information conveyed by each of the constituent STRFs. We next obtained a bicellular spike train, which consisted of the spikes in the peak of the cross-covariance function. We estimated an STRF for these synchronous spikes and calculated the information conveyed by the bicellular STRF, as well as the information for the original STRFs for each neuron in the pair. All information values were corrected for the number of spikes, because there were fewer spikes in the bicellular spike train than in either of the two constituent spike trains. To assess potential synchrony-based information gains, we compared the maximum of the information values of the two individual neurons in a pair with the information from the bicellular spikes (Fig. 7). Over the population, the bicellular information was significantly higher than for the single neuron information $(p<0.001$, signed-rank test; Fig. 7A). This result was recapitulated across all layers (Supra: $p=0.005$; Gran: $p<$ 0.001; Infra: $p=0.001$, signed-rank tests; Fig. $7 B-D$ ), indicating that the synchronous spikes carried more information than the spikes that were not synchronized.

The information gain of the synchronized spikes can be estimated using the ratio of the bicellular information compared with the maximum single neuron information. The information gain for bicellular spikes was approximately double that of the nonsynchronized spikes (population: $2.4 \pm 0.1$ bits/spike, mean \pm SE; Fig. $7 E$ ). The lowest gain was seen in Supra layers and the highest was seen in Infra layers (Supra: $1.7 \pm 0.3$; Gran: $2.1 \pm 0.2$; Infra: $2.5 \pm 0.1$ bits/spike, mean $\pm \mathrm{SE}$; Supra vs Infra: $p=0.029$; Gran vs Infra: $p=$ 0.018 , rank-sum tests; Fig. 7E). Further, information gains were seen for different cell types, with the highest gains for RSU-RSU pairs (FSU-RSU: $1.96 \pm 0.19$; RSU-RSU: $2.54 \pm 0.12$; FSU-FSU: $1.41 \pm$ 0.31 bits/spike, mean \pm SE; FSU-RSU vs RSU-RSU: $p=0.0084$, rank-sum test; Fig. $7 F$ ). These pairs had higher gains than FSU-RSU pairs, but all pairs had gains $>1$. 
Our results reveal significant property differences between columnar networks that are very local (i.e., mostly intralaminar) and more global (i.e., interlaminar). Local, short-distance functional connectivity $(\sim<150 \mu \mathrm{m})$ is characterized by sharp synchrony, higher correlation strengths, and high receptive field similarity (Fig. $8 B-G$ ). Longer-distance functional connectivity $(\sim>400 \mu \mathrm{m})$, by contrast, is less synchronous, has significantly lower correlation strength, and is related to receptive fields that are less similar than those within laminae (see Fig. $8 B-G$ for neuron separations $>150 \mu \mathrm{m})$. The nearly linear relationship between cortical distance and peak delay of the correlated activity (Fig. $8 D, E$ ) suggests an emphasis on global hierarchical processing. Further, the network activity within local circuits appears poised to encode and deliver stimulus information with high fidelity, because synchronous spikes carry nearly twice the information than the spikes of constituent neurons. These results suggest a special and distinct nature of local subnetworks within auditory cortex, with implications for information encoding and transmission. Sharp synchrony is a fundamental aspect of local auditory cortical processing and may allow for high-fidelity encoding and transmission of stimulus information to subsequent cortical and subcortical sites.

Our results showed that correlation strength decreases as the vertical distance between neurons increases (Fig. 8F). A similar trend has been observed in studies of horizontal interactions (Boucsein et al., 2011). This trend is puzzling, because intralaminar connections within a column may only account for $25 \%$ of a neuron's potential synapses (Binzegger et al., 2004; Boucsein et al., 2011); the other 75\% originate from outside the column. Even if column widths are considered to be 500 $\mu \mathrm{m}$, the number of synapses originating outside the column is still $>50 \%$ (Stepanyants et al., 2009). This trend appears to be a characteristic feature of cortical connectivity (Hellwig, 2000; Holmgren et al., 2003).

What accounts for the high correlation strengths between nearby neurons? Any account must be reconciled with anatomical constraints. Remarkably, the anatomical connection probabilities can be modeled accurately by making relatively few assumptions (Hellwig, 2000; Stepanyants and Chklovskii, 2005). The first assumption is that local connections are not specific, apart from geometrical constraints imposed by axons and dendrites. Second, when an axon and dendrite are in close proximity, they form a synapse. These two considerations produce accurate models of anatomical connectivity. However, merely
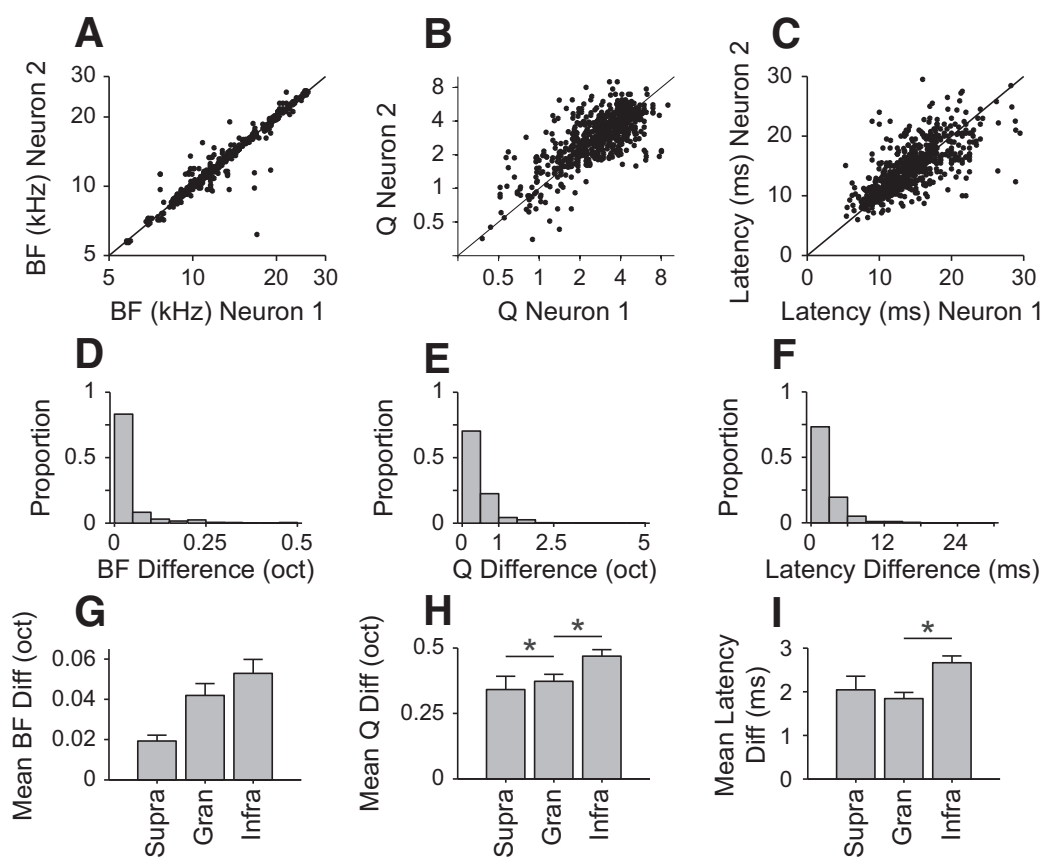

Figure 5. BF, spectral tuning, and latency for pairs of neurons. $A$, Comparison between BFs. For each data point, the value on the abscissa corresponds to the neuron in the pair with the shortest spike waveform duration. $\boldsymbol{B}$, Population BF differences, in oct. $C$, Mean BF difference versus layer. $\boldsymbol{D}, Q$ across the population of pairs. $\boldsymbol{E}$, Spectral tuning differences, in oct. $\boldsymbol{F}$, Spectral tuning differences across layers. $\boldsymbol{G}$, Latency of peak in STRF. $\boldsymbol{H}$, Latency differences. I, Latency differences versus layers. ${ }^{*} p<0.05 ;{ }^{* *} p<0.01$; ${ }^{* * *} p<0.001$.
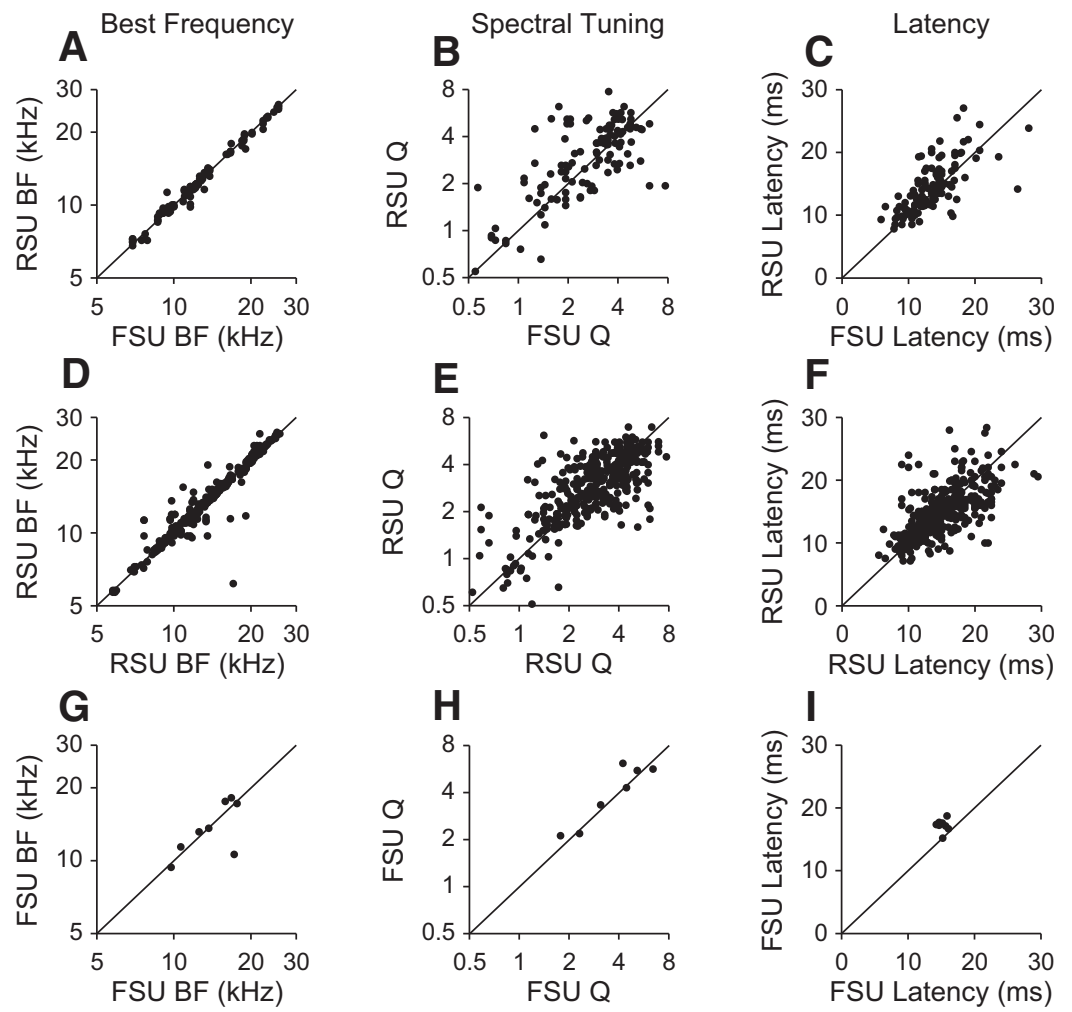

Figure 6. Comparison between $B F$, spectral tuning, and latency of neighboring auditory cortical neurons of differing cell types. Comparison between BF, $Q$, and latency for FSU-RSU pairs $(\boldsymbol{A}-\boldsymbol{C})$, RSU-RSU pairs $(\boldsymbol{D}-\boldsymbol{F})$, and FSU-FSU pairs $(\boldsymbol{G}-\boldsymbol{I})$.

counting synapses may not give a complete picture of the cortical features that influence local correlation strengths.

Additional characteristics of cortical processing must be considered to account for the higher correlation strengths of nearby 

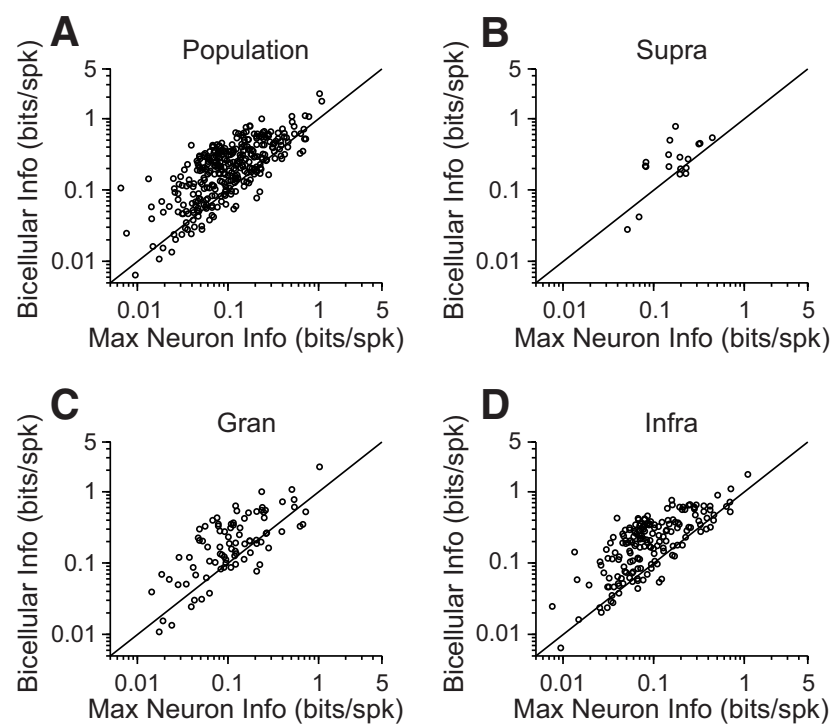

E

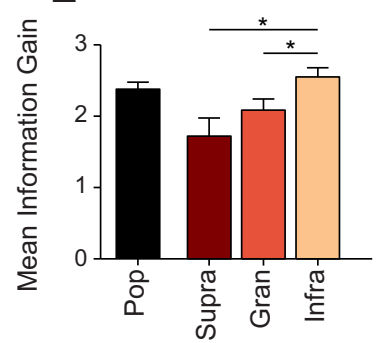

$\mathbf{F}$

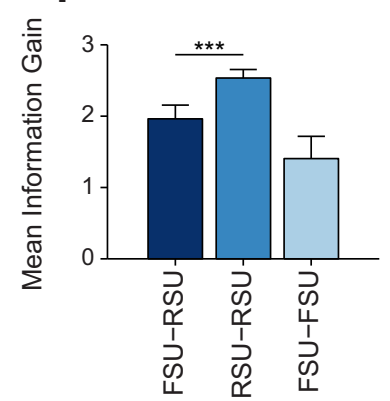

Figure 7. Bicellular information analysis. STRF information for synchronous spikes was compared with that for each neuron in a pair. $A$, Bicellular information (information for synchronous spikes for each pair of neurons) compared with the maximum of the information values for the neurons in the pair. $\boldsymbol{B}-\boldsymbol{D}$, Bicellular versus maximum neuron information for Supra $(\boldsymbol{B})$, Gran $(\boldsymbol{C})$, and Infra $(\boldsymbol{D})$ neurons. Bicellular information was higher for all comparisons (population: $p<0.001$; Supra: $p<0.005$, Gran: $p<0.001$; Infra: $p<0.001$; signed-rank tests). $\boldsymbol{E}$, Information gain (bicellular information/maximum single neuron information). $\boldsymbol{F}$, Information gain for cell-type pairs. ${ }^{*} p<0.05$; ${ }^{* *} p<0.01$; $^{* *} p<0.001$.

neurons. First, local neurons will have a greater probability of making multiple synaptic contacts with a nearby cell. Second, for pyramidal cells, these contacts will have their greatest influence locally, because basal dendrites sample a spherical volume encompassing the cell body and basal dendrites contain more dendritic area than apical dendrites (Larkman, 1991). Third, the timing of synaptic inputs from local neurons will show less temporal jitter, because the latencies of local neurons are similar. In comparison, the timing of inputs from distant neurons will not be as well coordinated, leading to decreased correlation strength. Finally, a consequence of the timing of inputs will be that spiketiming-dependent plasticity may strengthen inputs from nearby neurons. Together, these considerations may explain the sharp decline in correlation strength with increasing neuronal distance (Fig. $8 F$ ).

\section{Discussion}

Our investigation of relative response timing and receptive field properties of neurons with close spatial proximity in cat primary auditory cortex revealed five main principles of local subnetwork processing: (1) pairwise correlations of neighboring neurons show short delays and sharp synchrony, indicative of a functionally distinct and highly connective subnetwork; (2) cell-type

\begin{tabular}{|c|c|c|c|c|c|c|c|}
\hline \multirow[t]{2}{*}{ A } & A & \multicolumn{3}{|c|}{ Layer } & \multicolumn{3}{|c|}{ Cell Type } \\
\hline & Parameter & Supra & Gran & Infra & $F-R$ & $\mathrm{R}-\mathrm{R}$ & F-F \\
\hline & Peak Delay & - & - & - & - & - & - \\
\hline$\Phi$ & Half-Width & - & - & - & - & - & 0 \\
\hline & $\mathrm{CCC}$ & 0 & 0 & - & 0 & 0 & 0 \\
\hline 음 & CF Difference & - & - & - & - & - & - \\
\hline () & Q Difference & - & - & 0 & - & - & - \\
\hline \& & Latency Difference & e & - & 0 & - & - & - \\
\hline & RF Difference & - & - & 0 & - & - & - \\
\hline
\end{tabular}
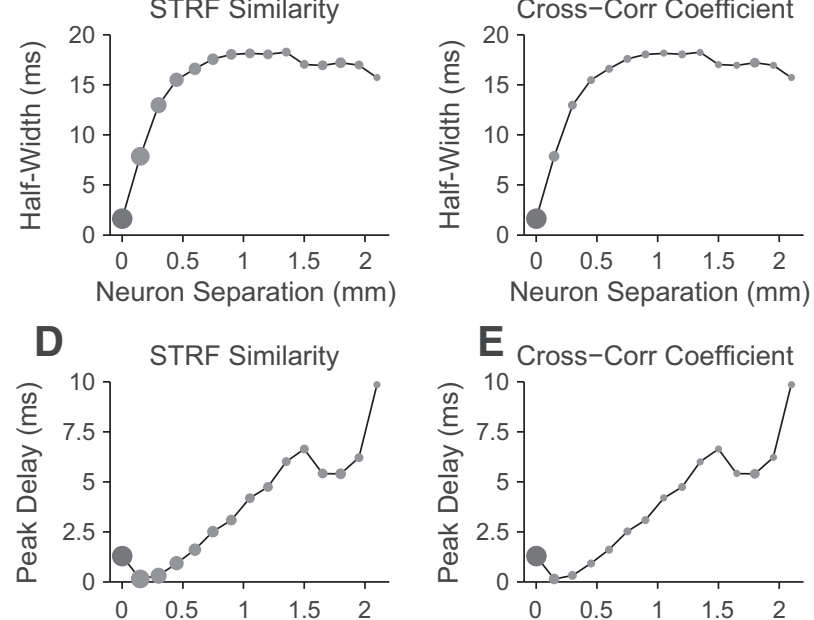

E Cross-Corr Coefficient
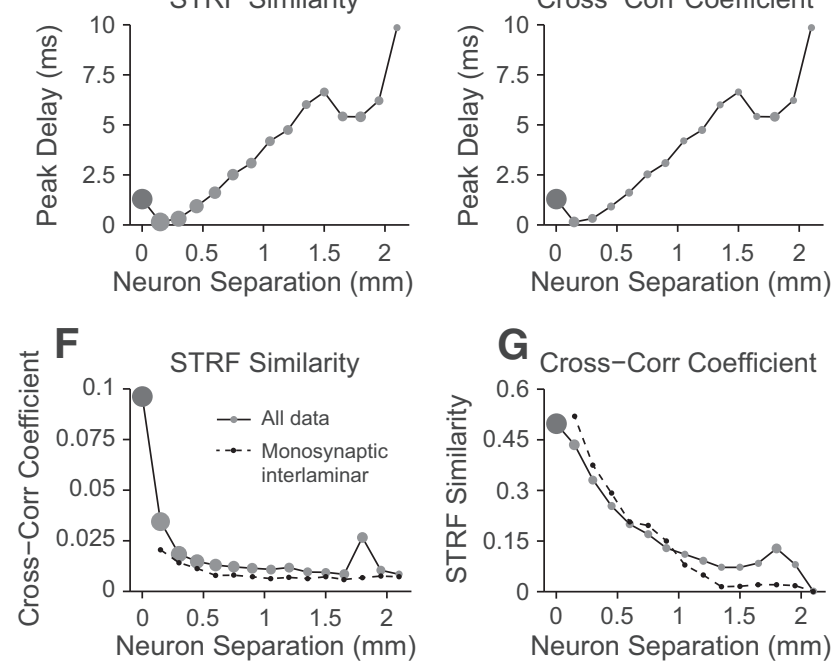

Figure 8. Summary of Al synchrony analysis. $\boldsymbol{A}$, Comparison of connectivity parameters between different layers (columns 2-4) or between cell type pairs (columns 5-7). F-R, FSURSU; R-R, RSU-RSU; F-F, FSU-FSU. RF difference $=1-$ STRF similarity. Circle size represents the relative magnitude of each parameter. Circles of different sizes represent significantly different values. Supra and Gran layers have closely matched laminar distributions. RSU-RSU pairs show the sharpest synchrony, whereas FSU-RSU pairs have the smallest receptive field differences. $\boldsymbol{B}-\boldsymbol{G}$, Bubble plots for synchrony and receptive field parameters for intralaminar (dark gray bubble, data from current study) and interlaminar (light gray bubbles; Atencio and Schreiner, 2010a) data. The title of each scatter plot indicates the parameter represented by the bubble. Bubble diameter is proportional to the parameter value. $\boldsymbol{B}, \mathrm{HW}$ versus neuron separation. The smallest HWs occur for neighboring neurons, and these pairs of neurons have the highest STRF similarity. C, HW versus neuron separation shows that $\mathrm{CCC}$ is strongest for nearby, synchronously spiking neurons. $\boldsymbol{D}, \boldsymbol{E}$, Peak delay versus neuron separation, with STRF similarity and CCC indicated by bubble size. $\boldsymbol{F}$, CCC versus neuron separation, with STRF similarity indicated by bubbles. G, STRF similarity versus neuron separation, with CCC indicated by bubbles. $\boldsymbol{F}$ and $\mathbf{G}$ also show data for putative monosynaptic interlaminar functional connectivity (dashed black lines).

composition (RSU, FSU) of the local pairs have no apparent influence on the local synchrony; (3) receptive field properties of neurons within synchronous subnetworks are highly similar, but 
not identical in all aspects, indicating that local neurons can perform different processing and that joint signaling may provide a more specific and powerful local network output; (4) local, celltype specific synchrony did not vary from layer to layer, but revealed a slightly higher synchrony in supragranular layers, so sharp synchrony is a general and defining characteristic of local cortical subcircuits within layers, in contrast to less synchronous interlaminar processing; and (5) synchronous spikes carry about twice the stimulus information of nonsynchronous spikes, indicating enhanced information processing compared with nonsynchronized events.

Previous studies in somatosensory cortex have shown that pairs of inhibitory neurons exhibit sharp firing synchrony (Swadlow et al., 1998; Hu et al., 2011). Whether they are electrically or chemically linked, inhibitory neurons fire in close concert in many sensory systems (Galarreta and Hestrin, 2002). We have shown that not only is this a feature of neighboring inhibitory neurons, but it also occurs for neighboring excitatory neurons and excitatory/inhibitory pairs in auditory cortex.

Synchrony in auditory cortex was characterized by very tight covariance functions that had peaks at delays $<4 \mathrm{~ms}$ indicative of monosynaptic functional connectivity or tight, concerted, common input. Although we cannot unambiguously determine anatomical connectivity, our results are consistent with findings that nearby neurons are highly interconnected (Holmgren et al., 2003; Fino and Yuste, 2011). This strong nearby connectivity was reflected in our data in the correlation strength between neurons with a cross-correlation coefficient that was on average $>0.07$. This intralaminar value is higher than the strengths for interlaminar correlation (Atencio and Schreiner, 2010a). Further, the correlation strength estimates are necessarily conservative and therefore have lower bounds due to the difficulties with unambiguously identifying action potentials from multiple neurons on the same electrode channel. Therefore, nearby AI neurons exhibit intralaminar functional connectivity that is markedly stronger than correlations between layers.

The synchrony we observed was present in layers 2 to 6 of auditory cortex and for all cell-type pairs, indicating preserved local processing principles. Synchrony and receptive field similarity of neighboring neurons was greatest in supragranular and granular layers and less so in infragranular layers. Therefore, supragranular layers may be capable of the most precise relative spike timing, enabling a more faithful projection of stimulus characteristics through their firing patterns. This may be a functional consequence of corticocortical connectivity patterns, which are dominated by remarkably precise and topographic supragranular projections (Lee and Winer, 2005). In contrast, infragranular pairs showed greater variability in response timing and receptive field properties but also conveyed the most information per synchronous spike. Layers 5 and 6 have the most heterogeneous connectivity patterns, projecting to cortical as well as thalamic, midbrain, and brainstem targets (Prieto and Winer, 1999; Winer and Prieto, 2001). The more diverse synchrony in infragranular layers may be a consequence of this diversity in projection targets (Mitani et al., 1985).

The synchrony for RSU-RSU pairs was higher than for the other cell-type pairs. Previously, it was noted that both electrical and chemical synapses lead to cortical synchrony between inhibitory-inhibitory pairs (Galarreta and Hestrin, 2002; Hu et al., 2011). Our results generalize these findings to other pairs of cell types that are connected through chemical synapses. Therefore, in auditory cortex, proximity is the greatest predictor of synchrony; cell type does not appear to be the main determining factor. Further, synchrony appears as a consequence of wiring patterns, with neighboring neurons more likely to be wired together, consistent with the view that connectivity is determined by the structure of local circuits and less so by microinfluences such as the composition of channels in a neuron (Hill et al., 2012).

\section{Functional processing}

Sharply synchronous firing of neighboring neurons was also indicative of similar receptive field properties. BF of neighboring neurons was highly conserved $(\Delta \mathrm{BF}<0.1 \mathrm{oct})$. The smallest $\mathrm{BF}$ differences were in supragranular layers, but overall the mean layer differences were small. This indicates that in the cat AI circuit, wiring is prioritized to conserve tonotopy. Other secondary parameters, such as spectral tuning and latency, were similar, but not to the same extent as BF. Consistent with earlier work in cats and guinea pigs, spectral tuning became less sharp in deeper layers, indicating broader frequency integration (Volkov and Galaziuk, 1989; Wallace and Palmer, 2008).

\section{Extrinsic and intrinsic correlation influences}

Synchronous firing may be a consequence of extrinsic and intrinsic influences. A possible significant extrinsic factor is stimulus correlations. When the correlations in the stimulus occur at the same timescale as those in the covariance function, it is difficult to discern whether functional connectivity is driven by stimulus or anatomical considerations. The dynamic ripple stimulus that we used had a maximum temporal modulation rate of $40 \mathrm{~Hz}$, or a period of $25 \mathrm{~ms}$. Synchronization of firing to the modulations in the stimulus would be expected to be maximal at the rising phase of the stimulus (i.e., $\sim 6.25 \mathrm{~ms}$ at $40 \mathrm{~Hz}$ ). Because crosscovariance functions had widths that were often $<2 \mathrm{~ms}$, stimulus correlations were not the main determinant of functional connectivity. Therefore, modulations in the stimulus are not expected to be the major determinant of functional connectivity. Furthermore, in this report and previously, we did not find evidence of significant stimulus synchronization when we estimated shift predictors. Finally, tightly aligned common input may also contribute functional connectivity, but this input would have to be more precise than the temporal features of the stimulus. The functional connectivity in the present study is unlikely to be dominated by stimulus driven influences (Atencio and Schreiner, 2010a).

Fluctuations in local field potentials (LFPs) may also influence neural spiking. LFP fluctuations occur over large cortical distances and may influence the joint firing of neurons through ephaptic coupling (Anastassiou et al., 2011). In ephaptic coupling, spiking probability occurs at specific phases of the LFP. However, ephaptic coupling is unlikely to cause the sharp synchrony we report. First, the DMR stimulus that we used desynchronizes the thalamocortical circuit (Miller and Schreiner, 2000). Second, ephaptic coupling acts over larger cortical distances. However, we found that within the same cortical column, intralaminar synchrony was markedly different from interlaminar functional connectivity. Third, ephaptic coupling acts over multiple milliseconds. However, the synchrony we observed is based on a subset of spikes that occur with submillisecond precision. These considerations make it unlikely that ephaptic coupling was a significant causal factor of the observed sharp synchrony.

Internal and external controls on our correlation analysis support the conclusion that the functional connectivity is related to intrinsic circuit properties. First, in a previous study, we esti- 
mated the relationship of the distance between neurons in a column and the peak delay in cross-covariance functions. Greater separations are expected to result in longer peak delays because an increase in synapse number and conduction distance will correspond to an increase in signal travel time. We found that the relationship between distance and peak delay corresponded to a propagation time of $0.22 \mathrm{~m} / \mathrm{s}$ within a column, which was similar to the propagation time found in slice $(0.26 \pm 0.05 \mathrm{~m} / \mathrm{s}$; Kubota et al., 1997; Atencio and Schreiner, 2010a). The similarity between the value that we obtained and the value from an independent and completely different preparation indicate that our cross-covariance functions are capable of capturing significant aspects of cortical anatomical connectivity. Second, our data and anatomical considerations reveal similar connection probabilities. We found that 535 of 670 pairs had significant functional connectivity (connection probability of 0.843 ). This compares favorably to estimates of connection probability from anatomical considerations (0.864; Braitenberg and Schüz, 1998). Therefore, the functional connectivity that we measured may be a reasonable reflection of the anatomical connectivity within local AI circuits.

In total, our results show that intralaminar local subnetworks contain neurons that fire synchronously and these synchronous spikes convey, on average, significantly more information about the stimulus than nonsynchronous spikes, which is indicative of their higher encoding fidelity.

\section{References}

Agmon A (2012) A novel, jitter-based method for detecting and measuring spike synchrony and quantifying temporal firing precision. Neural Syst Circuits 2:5. CrossRef Medline

Anastassiou CA, Perin R, Markram H, Koch C (2011) Ephaptic coupling of cortical neurons. Nat Neurosci 14:217-223. CrossRef Medline

Atencio CA, Schreiner CE (2008) Spectrotemporal processing differences between auditory cortical fast-spiking and regular-spiking neurons. J Neurosci 28:3897-3910. CrossRef Medline

Atencio CA, Schreiner CE (2010a) Columnar connectivity and laminar processing in cat primary auditory cortex. PLoS One 5:e9521. CrossRef Medline

Atencio CA, Schreiner CE (2010b) Laminar diversity of dynamic sound processing in cat primary auditory cortex. J Neurophysiol 103:192-205. CrossRef Medline

Atencio CA, Sharpee TO, Schreiner CE (2008) Cooperative nonlinearities in auditory cortical neurons. Neuron 58:956-966. CrossRef Medline

Atencio CA, Sharpee TO, Schreiner CE (2009) Hierarchical computation in the canonical auditory cortical circuit. Proc Natl Acad Sci U S A 106: 21894-21899. CrossRef Medline

Bandyopadhyay S, Shamma SA, Kanold PO (2010) Dichotomy of functional organization in the mouse auditory cortex. Nat Neurosci 13:361368. CrossRef Medline

Bannister AP, Thomson AM (2007) Dynamic properties of excitatory synaptic connections involving layer 4 pyramidal cells in adult rat and cat neocortex. Cereb Cortex 17:2190-2203. CrossRef Medline

Binzegger T, Douglas RJ, Martin KA (2004) A quantitative map of the circuit of cat primary visual cortex. J Neurosci 24:8441-8453. CrossRef Medline

Blomquist P, Devor A, Indahl UG, Ulbert I, Einevoll GT, Dale AM (2009) Estimation of thalamocortical and intracortical network models from joint thalamic single-electrode and cortical laminar-electrode recordings in the rat barrel system. PLoS Comput Biol 5:e1000328. CrossRef Medline

Boucsein C, Nawrot MP, Schnepel P, Aertsen A (2011) Beyond the cortical column: abundance and physiology of horizontal connections imply a strong role for inputs from the surround. Front Neurosci 5:32. CrossRef Medline

Braitenberg V, Schüz A (1998) Cortex: statistics and geometry of neuronal connectivity, Ed 2. New York: Springer.

Brosch M, Schreiner CE (1999) Correlations between neural discharges are related to receptive field properties in cat primary auditory cortex. Eur J Neurosci 11:3517-3530. CrossRef Medline
Bruno RM, Simons DJ (2002) Feedforward mechanisms of excitatory and inhibitory cortical receptive fields. J Neurosci 22:10966-10975. Medline

Chen C, Read HL, Escabí MA (2012) Precise feature based time scales and frequency decorrelation lead to a sparse auditory code. J Neurosci 32: 8454-8468. CrossRef Medline

Douglas RJ, Martin KA (2004) Neuronal circuits of the neocortex. Annu Rev Neurosci 27:419-451. CrossRef Medline

Eggermont JJ (1992) Neural interaction in cat primary auditory cortex. Dependence on recording depth, electrode separation, and age. J Neurophysiol 68:1216-1228. Medline

Eggermont JJ (2000) Sound-induced synchronization of neural activity between and within three auditory cortical areas. J Neurophysiol 83:2708 2722. Medline

Escabi MA, Schreiner CE (2002) Nonlinear spectrotemporal sound analysis by neurons in the auditory midbrain. J Neurosci 22:4114-4131. Medline

Fino E, Yuste R (2011) Dense inhibitory connectivity in neocortex. Neuron 69:1188-1203. CrossRef Medline

Galarreta M, Hestrin S (1999) A network of fast-spiking cells in the neocortex connected by electrical synapses. Nature 402:72-75. CrossRef Medline

Galarreta M, Hestrin S (2002) Electrical and chemical synapses among parvalbumin fast-spiking GABAergic interneurons in adult mouse neocortex. Proc Natl Acad Sci U S A 99:12438-12443. CrossRef Medline

Gibson JR, Beierlein M, Connors BW (1999) Two networks of electrically coupled inhibitory neurons in neocortex. Nature 402:75-79. CrossRef Medline

Halliday DM, Rosenberg JR (1999) Time and frequency domain analysis of spike train and time series data. In: Modern techniques in neuroscience research (Windhorst U, Johansson H, eds), pp 503-543. New York: Springer.

Hellwig B (2000) A quantitative analysis of the local connectivity between pyramidal neurons in layers $2 / 3$ of the rat visual cortex. Biol Cybern 82:111-121. CrossRef Medline

Hill SL, Wang Y, Riachi I, Schürmann F, Markram H (2012) Statistical connectivity provides a sufficient foundation for specific functional connectivity in neocortical neural microcircuits. Proc Natl Acad Sci U S A 109: E2885-2894. CrossRef Medline

Holmgren C, Harkany T, Svennenfors B, Zilberter Y (2003) Pyramidal cell communication within local networks in layer $2 / 3$ of rat neocortex. J Physiol 551:139-153. CrossRef Medline

Hu H, Ma Y, Agmon A (2011) Submillisecond firing synchrony between different subtypes of cortical interneurons connected chemically but not electrically. J Neurosci 31:3351-3361. CrossRef Medline

Imaizumi K, Schreiner CE (2007) Spatial interaction between spectral integration and frequency gradient in primary auditory cortex. J Neurophysiol 98:2933-2942. CrossRef Medline

Kawaguchi Y, Kubota Y (1993) Correlation of physiological subgroupings of nonpyramidal cells with parvalbumin- and calbindinD28k-immunoreactive neurons in layer V of rat frontal cortex. J Neurophysiol 70:387-396. Medline

Kawaguchi Y, Kubota Y (1997) GABAergic cell subtypes and their synaptic connections in rat frontal cortex. Cereb Cortex 7:476-486. CrossRef Medline

Kubota M, Sugimoto S, Horikawa J, Nasu M, Taniguchi I (1997) Optical imaging of dynamic horizontal spread of excitation in rat auditory cortex slices. Neurosci Lett 237:77-80. CrossRef Medline

Larkman AU (1991) Dendritic morphology of pyramidal neurones of the visual cortex of the rat: I. Branching patterns. J Comp Neurol 306:307319. CrossRef Medline

Lee CC, Winer JA (2005) Principles governing auditory cortex connections. Cereb Cortex 15:1804-1814. CrossRef Medline

Lewicki MS (1994) Bayesian modeling and classification of neural signals. Neural Comput 6:1005-1030. CrossRef

Lewicki MS (1998) A review of methods for spike sorting: the detection and classification of neural action potentials. Network 9:R53-78. CrossRef Medline

Markram H, Toledo-Rodriguez M, Wang Y, Gupta A, Silberberg G, Wu C (2004) Interneurons of the neocortical inhibitory system. Nat Rev Neurosci 5:793-807. CrossRef Medline

Miller LM, Schreiner CE (2000) Stimulus-based state control in the thalamocortical system. J Neurosci 20:7011-7016. Medline

Mitani A, Shimokouchi M, Itoh K, Nomura S, Kudo M, Mizuno N (1985) Morphology and laminar organization of electrophysiologically identi- 
fied neurons in the primary auditory cortex in the cat. J Comp Neurol 235:430-447. CrossRef Medline

Molotchnikoff S, Gillet PC, Shumikhina S, Bouchard M (2007) Spatial frequency characteristics of nearby neurons in cats' visual cortex. Neurosci Lett 418:242-247. CrossRef Medline

Otsuka T, Kawaguchi Y (2009) Cortical inhibitory cell types differentially form intralaminar and interlaminar subnetworks with excitatory neurons. J Neurosci 29:10533-10540. CrossRef Medline

Packer AM, Yuste R (2011) Dense, unspecific connectivity of neocortical parvalbumin-positive interneurons: a canonical microcircuit for inhibition? J Neurosci 31:13260-13271. CrossRef Medline

Prieto JJ, Winer JA (1999) Layer VI in cat primary auditory cortex: Golgi study and sublaminar origins of projection neurons. J Comp Neurol 404: 332-358. CrossRef Medline

Rosenberg JR, Amjad AM, Breeze P, Brillinger DR, Halliday DM (1989) The Fourier approach to the identification of functional coupling between neuronal spike trains. Prog Biophys Mol Biol 53:1-31. CrossRef Medline

Rothschild G, Nelken I, Mizrahi A (2010) Functional organization and population dynamics in the mouse primary auditory cortex. Nat Neurosci 13:353-360. CrossRef Medline

Schreiner CE (1991) Functional topographies in the primary auditory cortex of the cat. Acta Otolaryngol Suppl 491:7-15; discussion 16. Medline

Smith SL, Häusser M (2010) Parallel processing of visual space by neighboring neurons in mouse visual cortex. Nat Neurosci 13:1144-1149. CrossRef Medline

Stepanyants A, Chklovskii DB (2005) Neurogeometry and potential synaptic connectivity. Trends Neurosci 28:387-394. CrossRef Medline
Stepanyants A, Hirsch JA, Martinez LM, Kisvárday ZF, Ferecskó AS, Chklovskii DB (2008) Local potential connectivity in cat primary visual cortex. Cereb Cortex 18:13-28. CrossRef Medline

Stepanyants A, Martinez LM, Ferecskó AS, Kisvarday ZF (2009) The fractions of short- and long-range connections in the visual cortex. Proc Natl Acad Sci U S A 106:3555-3560. CrossRef Medline

Swadlow HA (2003) Fast-spike interneurons and feedforward inhibition in awake sensory neocortex. Cereb Cortex 13:25-32. CrossRef Medline

Swadlow HA, Beloozerova IN, Sirota MG (1998) Sharp, local synchrony among putative feed-forward inhibitory interneurons of rabbit somatosensory cortex. J Neurophysiol 79:567-582. Medline

Thomson AM, Lamy C (2007) Functional maps of neocortical local circuitry. Front Neurosci 1:19-42. CrossRef Medline

Volkov IO, Galaziuk AV (1989) Reactions of tonic-type neurons in the cat auditory cortex to tones of various frequency and intensity. Neurophysiology 21:498-506. Medline

Wallace MN, Palmer AR (2008) Laminar differences in the response properties of cells in the primary auditory cortex. Exp Brain Res 184:179-191. Medline

Winer JA, Prieto JJ (2001) Layer V in cat primary auditory cortex (AI): cellular architecture and identification of projection neurons. J Comp Neurol 434:379-412. CrossRef Medline

Wu GK, Arbuckle R, Liu BH, Tao HW, Zhang LI (2008) Lateral sharpening of cortical frequency tuning by approximately balanced inhibition. Neuron 58:132-143. CrossRef Medline 